\title{
Control of Large Vehicular Platoons: Improving Closed Loop Stability by Mistuning
}

\author{
Prabir Barooah Prashant G. Mehta João P. Hespanha
}

\begin{abstract}
We consider decentralized control of a platoon of $N$ identical vehicles moving in a straight line following a single lead vehicle moving at constant velocity. The control objective is for each vehicle to maintain the velocity of the leader and inter-vehicular separation using only the local information from itself and its two nearest neighbors. Each vehicle is modeled as a double integrator. To aid the analysis, we derive a continuous partial differential equation (PDE) approximation of the discrete platoon dynamics. The PDE model is used to explain the progressive loss of closed-loop stability with increasing number of vehicles, and to devise ways to combat this loss of stability.
\end{abstract}

If every vehicle uses the same controller, we show that the least stable closed-loop eigenvalue approaches zero as $O\left(\frac{1}{N^{2}}\right)$ in the limit of a large number $(N)$ of vehicles. We then show how to ameliorate this loss of stability by small amounts of "mistuning", i.e., changing the controller gains from their nominal values. We prove that with arbitrary small amounts of mistuning, the asymptotic behavior of the least stable closed loop eigenvalue can be improved to $O\left(\frac{1}{N}\right)$. These conclusions are validated for the discrete platoon via numerical calculations.

\section{INTRODUCTION}

We consider the problem of controlling a 1-dimensional platoon of $N$ identical vehicles such that individual vehicles move at a constant desired velocity $V_{d}$ with an intervehicular spacing of $\Delta$; shown schematically in Figure 1(a). Due to its relevance to an automated highway system, this problem has been extensively studied in literature [1-3]. A controlled vehicular platoon with a constant but small intervehicular distance can improve the capacity of a highway.

In this paper, we consider a decentralized bidirectional control architecture: the control action at a vehicle depends upon its own velocity and the relative position errors between itself and its nearest neighbors (the vehicles immediately ahead and behind it). Decentralized refers to the constraint that a vehicle is allowed to use only its local information, and "bidirectional" refers to the feature whereby information from both the vehicles ahead (predecessor) and behind (follower) is used. In contrast, a centralized control architecture would require information from all the vehicles to be transmitted to a central controller,

Prabir Barooah and João P. Hespanha are with the Dept. of Electrical and Computer Engg., Univ. of California, Santa Barbara, and their work is supported by the Institute for Collaborative Biotechnologies through the ARO grant DAAD19-03-D-0004 and by the NSF Grant ECS-0242798. Prshant G. Mehta is with the Dept. of Mechanical Science and Engg., Univ. of Illinois, Urbana-Champaign, IL, and his work is supported by NSF grant CMS 05-56352.. making the communication overhead impractical for large platoons. Since the vehicles are assumed to be identical, one particularly important special case is the so-called symmetric bidirectional control, where all vehicles use identical controllers that are furthermore symmetric with respect to the predecessor and the follower position errors.

Control of large vehicular platoons with a desired constant inter-vehicular spacing is challenging due to "string instability" or "slinky-type effects" referring to the amplification of disturbance as it propagates through the platoon. Here, the effect of disturbance felt by one vehicle gets amplified for vehicles both ahead and behind it, and the amplification grows without bound as the number of vehicles increases [2, 4-6].

These effects are essentially due to the progressive loss of closed-loop damping as the number of vehicles increases. Jovanović et. al. have recently considered an LQR formulation of the platoon problem [1]. They showed that the eigenvalues of the closed-loop approach the imaginary axis as the number of vehicles in the platoon increases without bound. Note that an LQR formulation will in general lead to a centralized control architecture.

This paper is concerned with the analysis of closedloop instabilities but more importantly with methods for their amelioration while using decentralized bi-directional architectures. There are three contributions of this work that are summarized below. In order to facilitate the analysis, we derive a linear partial differential equation (PDE) continuous model from the discrete platoon dynamics. Note that "discrete" here refers to the spatial discreteness, the time axis is continuous in both the platoon dynamics and the PDE model. The results of this paper are obtained by analyzing the PDE; they are then validated by numerical computation of the closed loop platoon dynamic equations. The PDE model is inspired by the extensive literature on traffic dynamics; cf. [7] and references therein. In the limit of large $N$, the vehicles moving in a straight line are idealized as particles in a 1-dimensional flow.

The second contribution of this paper is to use the PDE model to show that the least stable closed-loop eigenvalue of the discrete platoon dynamics approaches zero as $O\left(\frac{1}{N^{2}}\right)$, where $N$ is the number of vehicles. This conclusion is independent to the choice of controller gains.

The third contribution and the most significant advantage of using a PDE based analysis is that the PDE reveals, better 


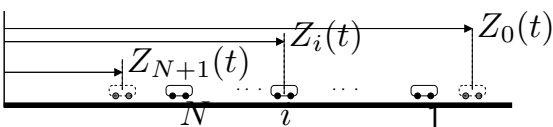

(a) A platoon with fictitious lead and follow vehicles.

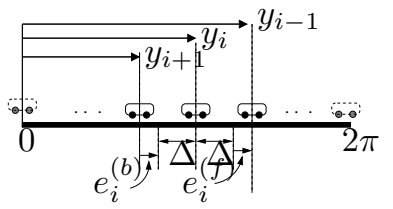

(b) Same platoon in $y$ coordinates

Fig. 1. A platoon with $N$ vehicles moving in one dimension.

than the discrete equations do, the reason for the loss of stability and suggests "mistuning" to improve the stability margin. In particular, assignment of controller gains such that a vehicle utilizes information from the preceding vehicle differently from the following vehicle is seen to be beneficial. Our main result Corollary 2 shows how to achieve the best improvement in closed-loop stability by exploiting this asymmetry. In particular, we show that an arbitrary small perturbation (asymmetry) in the controller gains from their nominal (symmetric) value can improve the closed-loop damping such that the least stable eigenvalue now approaches 0 only as $O\left(\frac{1}{N}\right)$. Direct computation of eigenvalues in discrete platoons is used to validate these results. The mistuning profile is determined by optimizing the smallest eigenvalue using a perturbation-based method from [8]. We note that the mistuning based approaches have been used for stability augmentation in many structural applications; see [9] for a recent references.

The rest of the paper is organized as follows: section II describes the platoon problem formulation; section III then describes the derivation of the PDE model. In section IV the PDE is analyzed to explain the loss of stability with $N$, and section $\mathrm{V}$ describes how to ameliorate such loss of stability by mistuning.

\section{Problem Statement}

Consider a platoon of $N$ identical vehicles moving in a straight line as shown schematically in Figure 1(a). Let $Z_{i}(t)$ and $V_{i}(t)$ denote the position and the velocity, respectively, of the $i^{\text {th }}$ vehicle for $i=1,2, \ldots, N$. Since the inter-connected platoon dynamics are of primary interest, a simple double integrator is used to model the essential dynamics of an individual vehicle:

$$
\ddot{Z}_{i}=U_{i}
$$

where $U_{i}$ is the control (engine torque) applied on the $i^{\text {th }}$ vehicle. Formally, such a model arises after the velocity dependent drag and other non-linear terms have been eliminated by using feedback linearization $[3,6]$. The control objective is to maintain a constant inter-vehicular distance $\Delta$ and a constant velocity $V_{d}$ for every vehicle. The control architecture is required to be decentralized, so that every vehicle can use locally available measurements only.

Following [1], we introduce a fictitious lead vehicle and a fictitious follow vehicle, indexed as 0 and $N+1$ respectively. Their behavior is specified by imposing constant velocity trajectories as $Z_{0}(t)=V_{d} t$ and $Z_{N+1}=$ $V_{d} t-(N+1) \Delta$. Now every vehicle in the platoon, including the $1^{\text {st }}$ and the $N^{\text {th }}$ vehicle, has two neighbors and can employ a decentralized bidirectional control architecture.

To facilitate the analysis, consider a coordinate change

$$
y_{i}=2 \pi\left(\frac{Z_{i}(t)-V_{d} t+L}{L}\right), \quad v_{i}=2 \pi \frac{V_{i}-V_{d}}{L},
$$

where $L$ denotes the platoon length, which equals $(N+1) \Delta$ in scenario I and $N \Delta$ in scenario II. Figure 1(b) depicts the schematic of the platoon in the new coordinates. The normalization ensures that $y_{0}(t) \equiv 2 \pi, y_{i}(t) \in[0,2 \pi]$, and $y_{N+1}(t) \equiv 0$. Here, we have implicitly assumed that the deviations of the vehicle positions and velocities from their desired values are small.

In the normalized coordinates, the dynamics of the $i^{\text {th }}$ vehicle are described by

$$
\ddot{y}_{i}=u_{i},
$$

where $u_{i}:=2 \pi U_{i} / L$. The desired spacing and velocities are

$$
\delta:=\frac{\Delta}{L / 2 \pi}, \quad v_{d}:=\frac{V_{d}-V_{d}}{L / 2 \pi}=0,
$$

and the desired position of the $i^{\text {th }}$ vehicle is

$$
y_{i}^{d}(t) \equiv 2 \pi-i \delta
$$

The position and velocity errors for the $i^{\text {th }}$ vehicle in the $y$ coordinate are:

$$
\tilde{y}_{i}(t)=y_{i}(t)-y_{i}^{d}(t), \quad \tilde{v}_{i}=v_{i}-v_{d}=v_{i} .
$$

We note that $\tilde{v}_{0}=\tilde{v}_{N+1}=0$ for the fictitious lead and follow vehicles.

For the purposes of control, it is useful to introduce the front and back relative (position) errors for the $i^{\text {th }}$ vehicle:

$$
\begin{gathered}
e_{i}^{(f)}=\frac{Z_{i-1}-Z_{i}-\Delta}{L / 2 \pi}=y_{i-1}-y_{i}-\delta, \\
e_{i}^{(b)}=\frac{Z_{i}-Z_{i+1}-\Delta}{L / 2 \pi}=y_{i}-y_{i+1}-\delta,
\end{gathered}
$$

for $i=1, \ldots, N$. The quantity $e_{i}^{(f)}$ denotes the front relative position error between the $i^{\text {th }}$ and its predecessor $(i-1)$ vehicle, and $e_{i}^{(b)}$ denotes the back relative position error between the $i^{\text {th }}$ and its follower $(i+1)$ vehicle. The relative errors, including the velocity error, can be obtained by on-board devices such as radars, GPS and speed sensors. Consistent with the decentralized bidirectional linear control architecture, the control $u_{i}$ for the $i^{\text {th }}$ vehicle is assumed to depend only on 1 ) its velocity $\tilde{v}_{i}$, and 2) the relative position errors between itself and its immediate neighbors. That is,

$$
u_{i}=k_{i}^{(f)} e_{i}^{(f)}-k_{i}^{(b)} e_{i}^{(b)}-b_{i} \tilde{v}_{i}
$$


where $k_{i}^{(\cdot)}, b_{i}$ are positive constants. The first two terms are used to compensate for any deviation away from nominal with the predecessor and the follower vehicles respectively. The third term is used to obtain a zero steady-state error in velocity. In principle, relative velocity errors between neighboring vehicles can also be incorporated into the control, but we do not examine this situation here.

To describe the closed-loop dynamics of the platoon, define

$$
\begin{aligned}
\tilde{\mathbf{y}} & :=\left[\tilde{y}_{1}, \tilde{y}_{2}, \ldots, \tilde{y}_{N}\right]^{T}, \\
\tilde{\mathbf{v}} & :=\left[\tilde{v}_{1}, \ldots, \tilde{v}_{N}\right]^{T} .
\end{aligned}
$$

For scenario I with fictitious lead and follow vehicles, the control law (3) yields the following closed loop dynamics.

$$
\left[\begin{array}{c}
\dot{\tilde{\mathbf{y}}} \\
\dot{\tilde{\mathbf{v}}}
\end{array}\right]=\underbrace{\left[\begin{array}{cc}
0 & I \\
-K_{\mathrm{I}}^{(f)} M^{T}-K_{\mathrm{I}}^{(b)} M & -B
\end{array}\right]}_{A_{L-F}}\left[\begin{array}{c}
\tilde{\mathbf{y}} \\
\tilde{\mathbf{v}}
\end{array}\right]
$$

where $K_{\mathrm{I}}^{(f)}=\operatorname{diag}\left(k_{1}^{(f)}, k_{2}^{(f)}, \ldots, k_{N}^{(f)}\right), K_{\mathrm{I}}^{(b)}=$ $\operatorname{diag}\left(k_{1}^{(b)}, k_{2}^{(b)}, \ldots, k_{N}^{(b)}\right), B=\operatorname{diag}\left(b_{1}, b_{2}, \ldots, b_{N}\right)$ and

$$
M=\left[\begin{array}{cccccc}
1 & -1 & 0 & \ldots & & \\
0 & 1 & -1 & & & \\
\vdots & & & \ddots & & 0 \\
& & & & 1 & -1 \\
& & & \ldots & 0 & 1
\end{array}\right] .
$$

Our goal is to understand the progressive loss of closed loop stability with increasing $N$ and to devise ways to ameliorate such a loss by appropriately choosing the controller gains. While in principle this can be done by analyzing the eigenvalues of the matrix $A_{L-F}$, we approximate the dynamics of the discrete platoon by a partial differential equation (PDE) which we then use for analysis and control design.

\section{Continuous Model of Vehicle Platoon}

In this section, we develop a continuous PDE approximation of the (spatially) discrete platoon dynamics. The PDE is derived with respect to a normalized spatial coordinate $x \in[0,2 \pi]$. We recall that the normalized location of the $i^{\text {th }}$ vehicle (denoted as $y_{i}$ ) too was defined with respect to this coordinate system. In effect, the two symbols $x$ and $y$ correspond to the same coordinate representation but are used here to distinguish the continuous and discrete formulations.

With respect to the normalized coordinate, every car is nominally assumed to lie within an interval of length $\delta$ (see Fig. 1(b)). For the purpose of a continuous approximation, we smear each vehicle over its interval to get a constant mean density

$$
\rho_{0} \approx \frac{N}{2 \pi}=\frac{1}{\delta}
$$

for $N$ vehicles in the platoon. The dynamics of the individual vehicles in the platoon create perturbations in the density, with the local density $\rho(x, t)$ increasing (decreasing) as the cars move closer (apart). The starting point of macroscopic continuous models of traffic flow thus is the continuity equation, which relates the density $\rho(x, t)$ (vehicles per unit characteristic length) at spatial coordinate $x \in[0,2 \pi]$ and time $t \in[0, \infty)$ with the velocity $v(x, t):$

$$
\frac{\partial \rho}{\partial t}+\frac{\partial(\rho v)}{\partial x}=0
$$

In order to analyze small perturbations about the mean, we define the perturbed quantities $\tilde{\rho}, \tilde{v}$ by the relations

$$
\rho(x, t)=\rho_{0}+\tilde{\rho}(x, t), \quad v(x, t)=0+\tilde{v}(x, t),
$$

where the mean velocity is zero because of our choice of the coordinate system (see (1)). Even though $v$ and $\tilde{v}$ are the same, we use $\tilde{v}$ to draw attention to the fact that the velocity is a small perturbation of the mean value. For such perturbations, the linearized continuity equation is given by

$$
\frac{\partial \tilde{\rho}}{\partial t}+\rho_{0} \frac{\partial \tilde{v}}{\partial x}=0 \Rightarrow \frac{\partial \tilde{v}}{\partial x}=-\frac{1}{\rho_{0}} \frac{\partial \tilde{\rho}}{\partial t}
$$

This equation is consistent with the physical intuition whereby a positive gradient in velocity (due to say the predecessor speeding up or the follower slowing down) will cause the local density to decrease. In order to study density perturbations, one thus needs to specify the velocity which arises due to the linearized momentum balance:

$$
\frac{\partial v}{\partial t}=F(x, t) \Rightarrow \frac{\partial \tilde{v}}{\partial t}=u(x, t),
$$

where $F(x, t)$ is the acceleration due to the control $u(x, t)$ and possible disturbances. Here, we focus only on the control. Using (3), the control for the $i^{\text {th }}$ vehicle in the platoon is of the form:

$$
\begin{aligned}
u_{i}(t) & =\underbrace{u_{i}^{(p f)}(t)-u_{i}^{(p b)}(t)}_{u_{i}^{(p)}(t)}+u_{i}^{(v)}(t), \quad \text { where } \\
u_{i}^{(p f)}(t) & :=k_{i}^{(f)}\left(y_{i-1}(t)-y_{i}(t)-\delta\right), \\
u_{i}^{(p b)}(t) & :=k_{i}^{(b)}\left(y_{i}(t)-y_{i+1}(t)-\delta\right), \\
u_{i}^{(v)}(t) & :=-b \tilde{v}_{i}(t) .
\end{aligned}
$$

where $u_{i}^{(p f)}, u_{i}^{(p b)}$ are the position dependent $f$ ront and $b$ ack control terms. Corresponding to this discrete control law, we derive a continuous approximation

$$
u(x, t)=\underbrace{u^{(p f)}(x, t)-u^{(p b)}(x, t)}_{u^{(p)}(x, t)}+u^{(v)}(x, t),
$$

such that $u^{(p f)}\left(y_{i}, t\right)=u_{i}^{(p f)}(t), u^{(p b)}\left(y_{i}, t\right)=u_{i}^{(p b)}(t)$, and $u^{(v)}\left(y_{i}, t\right)=u_{i}^{(v)}(t)$. Now,

$$
\begin{aligned}
u_{i}^{(p f)} & =k_{i}^{(f)}\left(y_{i-1}-y_{i}-\delta\right)=k_{i}^{(f)}\left(1-\frac{\delta}{y_{i-1}-y_{i}}\right)\left(y_{i-1}-y_{i}\right) \\
& \approx \int_{y_{i}}^{y_{i-1}} k_{p f}(x)\left(1-\frac{\rho(x)}{\rho_{0}}\right) d x,
\end{aligned}
$$

where the approximation is obtained by smearing the control action over the interval $\left[y_{i}, y_{i-1}\right]$ and substituting $k_{f}(x)$ for the discrete control gain $k_{i}^{(f)}$. Since $\rho=\rho_{0}+\tilde{\rho}$, we have $u_{i}^{(p f)}(t) \approx-\frac{1}{\rho_{0}} \int_{y_{i}}^{y_{i-1}} k_{f}(x) \tilde{\rho}(x, t) d x=-\frac{1}{\rho_{0}}\left[k_{f} \tilde{\rho}\right]\left(x^{+}, t\right) \delta$, 
by the Mean Value Theorem, where $x^{+} \in\left[y_{i}, y_{i-1}\right]$. Since $u_{i}^{(p f)}(t)=u^{(p f)}\left(y_{i}, t\right)$ and $\delta=1 / \rho_{0}$ (see (5)), we take

$$
\begin{aligned}
& u^{(p f)}(x, t)=-\frac{1}{\rho_{0}^{2}}\left[\begin{array}{ll}
k_{f} & \tilde{\rho}
\end{array}\right]\left(x^{+}, t\right), \\
& u^{(p b)}(x, t)=-\frac{1}{\rho_{0}^{2}}\left[k_{b} \tilde{\rho}\right]\left(x^{-}, t\right),
\end{aligned}
$$

where $x^{+} \in\left[y_{i}, y_{i-1}\right]$ and $x^{-} \in\left[y_{i+1}, y_{i}\right]$. Using (8),

$$
\begin{aligned}
u^{(p)}(x, t) & =u^{(p f)}(x, t)-u^{(p b)}(x, t) \\
& =-\frac{1}{\rho_{0}^{2}}\left(\left[\begin{array}{ll}
k_{f} & \tilde{\rho}
\end{array}\right]\left(x^{+}, t\right)-\left[\begin{array}{ll}
k_{b} & \tilde{\rho}
\end{array}\right]\left(x^{-}, t\right)\right) .
\end{aligned}
$$

In order to specify the control, one thus needs to approximate the terms on the right hand side as functions of $(x, t)$. For a small perturbation about a nominally symmetric bidirectional architecture, a valid approximation is obtained by taking $x^{+}-x^{-} \approx \delta$ which yields

$$
\begin{aligned}
u^{(p)}(x, t) & \approx-\frac{1}{\rho_{0}^{2}}\left[\left[k_{p}^{(-)} \tilde{\rho}\right](x, t)+\frac{\delta}{2} \frac{\partial\left[k^{(+)} \tilde{\rho}\right]}{\partial x}(x, t)\right] \\
& =-\frac{1}{\rho_{0}^{2}} k^{(-)} \tilde{\rho}-\frac{1}{2 \rho_{0}^{3}} \frac{\partial}{\partial x}\left(k^{(+)} \tilde{\rho}\right),
\end{aligned}
$$

where

$$
k^{(+)}:=k_{f}(x)+k_{b}(x), \quad k^{(-)}:=k_{f}(x)-k_{b}(x) .
$$

The velocity feedback term $u_{i}^{(v)}$ has a continuous counterpart $u^{(v)}(x, t)=-b(x) \tilde{v}(x, t)$. With the feedback control $u(x, t)=u^{(p)}(x, t)+u^{(v)}(x, t)$, where $u^{(p)}(x, t)$ is given by (9), the linearized momentum equation (7) becomes

$$
\frac{\partial \tilde{v}}{\partial t}=-\left[\frac{1}{\rho_{0}^{2}} k^{(-)} \tilde{\rho}+\frac{1}{2 \rho_{0}^{3}} \frac{\partial}{\partial x}\left(\tilde{\rho} k^{(+)}\right)+b \tilde{v}\right] .
$$

Upon differentiating both sides with respect to $t$ and using the continuity equation (6) we obtain the PDE that describes small velocity perturbations $\tilde{v}(x, t)$ of the platoon:

$$
\left(\frac{\partial^{2}}{\partial t^{2}}+b \frac{\partial}{\partial t}\right) \tilde{v}=\frac{1}{\rho_{0}}\left(k_{p}^{(-)} \frac{\partial \tilde{v}}{\partial x}\right)+\frac{1}{2 \rho_{0}^{2}} \frac{\partial}{\partial x}\left(k_{p}^{(+)} \frac{\partial \tilde{v}}{\partial x}\right)
$$

Because of the fictitious lead and follow vehicles, the appropriate boundary conditions are of the Dirichlet type:

$$
\tilde{v}(0, t)=\tilde{v}(2 \pi, t)=0 . \quad \forall t \in[0, \infty)
$$

\section{ANALYSIS OF LOSS OF STABILITY}

In this section we analyze how the least stable eigenvalue of the PDE model of the vehicular platoon depends on $N$. We explain the loss of closed loop stability of the platoon as $N \rightarrow \infty$ by showing that the least stable eigenvalue of the PDE approaches 0 as $O\left(\frac{1}{N^{2}}\right)$ no matter how the controller gains are chosen as long as they are the same for all the vehicles. These predictions are later validated by direct computation of eigenvalues of $A_{L-F}$. To this end, we consider the case when all the control gains are constant: $k_{p f}(x)=k_{p b}(x) \equiv k_{0}$ and $b(x) \equiv b_{0}$. The governing PDE (11) simplifies to

$$
\left(\frac{\partial^{2}}{\partial t^{2}}+b_{0} \frac{\partial}{\partial t}-a_{0}^{2} \frac{\partial^{2}}{\partial x^{2}}\right) \tilde{v}=0, \quad a_{0}^{2}:=\frac{k_{0}}{\rho_{0}^{2}},
$$

which is a damped wave equation, with $a_{0}$ being the wave speed. In order to compute the eigenvalues of the PDE, we take the Laplace transform of (13) w.r.t. to time $t$ :

$$
s^{2} q(x, s)+b_{0} s q(x, s)-a_{0}^{2} \frac{d^{2}}{d x^{2}} q(x, s)=0,
$$

where $q(x, s)$ denotes the Laplace transform of $\tilde{v}(x, t)$. This equation can be written as

$$
\frac{d^{2} q}{d x^{2}}=\lambda q(x), \quad \lambda:=\frac{s^{2}+b_{0} s}{a_{0}^{2}},
$$

which is an eigenvalue problem involving the Laplacian. The next lemma summarizes how the eigenvalues of the PDE depend of the parameters of the problem. The lemma can be verified by using the fact that $\sin \left(\frac{l x}{2}\right)$ is an eigenfunction of the Laplacian in (15) and that $\lambda_{l}=-\frac{l^{2}}{4}$ is the corresponding eigenvalue, for every positive integer $l$.

Lemma 1: Consider the eigenvalue problem (15) for the linear partial differential equation (13) with Dirichlet boundary conditions. The $l^{\text {th }}$ eigenvalue pair, $l=1,2, \ldots$, is given the analytical formula

$$
s_{l}^{ \pm}=\frac{-b_{0} \pm \sqrt{b_{0}^{2}-a_{0}^{2} l^{2}}}{2} .
$$

For large values of $N, \rho_{0} \approx \frac{N}{2 \pi}$, therefore $a_{0}^{2} l^{2} \approx$ $4 \pi^{2} k_{0} \frac{l^{2}}{N^{2}}$. We look for $l$ such that $a_{0}^{2} l^{2}<b_{0}^{2}$, i.e.,

$$
l<\frac{b_{0} N}{2 \pi \sqrt{k_{0}}}=: l_{c},
$$

which makes the discriminant $\left(b_{0}^{2}-a_{0}^{2} l^{2}\right)>0$, and correspondingly, the eigenvalues $s_{l}^{ \pm}$real. When $s_{l}^{ \pm}$are real, the eigenvalue $s_{l}^{+}$is closer to the origin than $s_{l}^{-}$; so we call $s_{l}^{+}$the less stable $l^{\text {th }}$ eigenvalue. The following corollary to Lemma 1, which is straightforward to prove, shows the dependence of the less stable $l^{\text {th }}$ eigenvalue on $N$.

Corollary 1: Consider the eigenvalue problem for the linear partial differential equation (13) with Dirichlet boundary conditions. The $l^{t h}$ less stable eigenvalue $s_{l}^{+}$ depends on $N$ as

$$
s_{l}^{+}=-\frac{\pi^{2} k_{0}}{b_{0}} \frac{l^{2}}{N^{2}}+O\left(\frac{1}{N^{4}}\right), \quad \forall l<<l_{c},
$$

where $l_{c}$ is defined in (16).

The corollary shows that the least stable eigenvalue is

$$
s_{1}^{+} \approx-\frac{\pi^{2} k_{0}}{b_{0}} \frac{1}{N^{2}}
$$

for large values of $N$. Therefore, to keep the least stable eigenvalue bounded away from 0 using a constant position feedback gain $k_{0}$, one has to increase the control gain $k_{0}$ as $N^{2}$. Jovanovic et. al. studied the LQR control of a platoon on a circle [1] and showed that to keep the closed loop eigenvalues bounded away from zero, one has to increase the control gains as $N^{2}$. Although the LQR solution in general may not lead to a decentralized architecture, both formulations seem to suffer from similar fundamental limitations. validation of prediction in (17) is provided in the next section - together with a way to combat this loss of stability. 


\section{REDUCING LOSS OF STABILITY BY MISTUNING}

In this section we study how to increase the damping of the least stable eigenvalue, i.e., increase the magnitude of its real part, by making the front and back position feedback gains $k_{f}$ and $k_{b}$ suitable functions of $x$. We will add a small perturbation, or "mistuning", on top of the nominal values of the control gains, and study the effect of this mistuning on the eigenvalues of the linearized PDE (11). The mistuning profile is determined by optimizing the smallest eigenvalue using a perturbation based method from [8].

Consider the position feedback gain profiles:

$$
k_{f}(x)=k_{0}+\epsilon k_{f}^{a}(x), \quad k_{f}(x)=k_{0}+\epsilon k_{b}^{a}(x),
$$

where $\epsilon>0$ is a small number and $k_{f}^{a}(x), k_{b}^{a}(x) \in \mathcal{L}^{2}$ are functions that determine the mistuning of the gains. Define

$$
k_{s}:=k_{f}^{a}(x)+k_{b}^{a}(x), \quad k_{m}:=k_{f}^{a}(x)-k_{b}^{a}(x),
$$

so that

$$
k_{p}^{(+)}(x)=2 k_{0}+\epsilon k_{s}(x), \quad k_{p}^{(-)}(x)=\epsilon k_{m}(x) .
$$

The mistuned version of the PDE (11) becomes

$$
\frac{\partial^{2} \tilde{v}}{\partial t^{2}}+b_{0} \frac{\partial \tilde{v}}{\partial t}-a_{0}^{2} \frac{\partial^{2} \tilde{v}}{\partial x^{2}}=\epsilon\left[\frac{1}{2 \rho_{0}^{2}} \frac{\partial^{2}\left(k_{s} \tilde{v}\right)}{\partial x^{2}}+\frac{1}{\rho_{0}} \frac{\partial\left(k_{m} \tilde{v}\right)}{\partial x}\right]
$$

Our interest is in designing the mistuning profiles $k_{s}(x)$ and $k_{m}(x)$ to reduce the movement of the least stable eigenvalue towards 0 with increasing $N$, and in achieving this with small mistuning amplitude $\epsilon$. It turns out that a vast improvement in the closed loop damping is possible by vanishingly small amounts of mistuning when the mistuning profile $k_{m}(x)$ is chosen appropriately; $k_{s}$ does not play as important a role as $k_{m}$ does. The following theorem provides analytical expressions on the $k$ th less stable eigenvalues of the governing PDE with mistuning.

Theorem 1: Consider the mistuning gain profiles (18) with $k_{0 f}=k_{0 b}=k_{0}$ so that the governing PDE is given by (20). The less stable $l^{\text {th }}$ eigenvalue of the mistuned PDE (20) with Dirichlet boundary conditions is given by:

$s_{l}^{+}(\epsilon)=\frac{\epsilon l}{2 b_{0} N} \int_{0}^{2 \pi} k_{m}(x) \sin (l x) d x+O\left(\epsilon^{2}\right)+O\left(\frac{1}{N^{2}}\right)$,

which is valid for each $l$ as $\epsilon \rightarrow 0$ and $N \rightarrow \infty$.

The usefulness of this result arises because it provides a means to add damping to the $l^{\text {th }}$ less stable eigenvalue $s_{l}^{+}$by suitably choosing the mistuning profile $k_{m}(x)$. The following corollary, which follows from Theorem 1 trivially, shows how the least stable eigenvalue depends on $N$ when the "optimal" mistuning pattern is applied, no matter how small the mistuning amplitude $\epsilon$ is.

Corollary 2: Consider the problem of minimizing the least-stable eigenvalue of the PDE (20) with Dirichlet boundary condition (12) by choosing a function $k_{m}(x) \in$ $L^{2}([0,2 \pi])$ such that $\int_{0}^{2 \pi}\left|k_{m}(x)\right|^{2} d x=1$. In the limit as

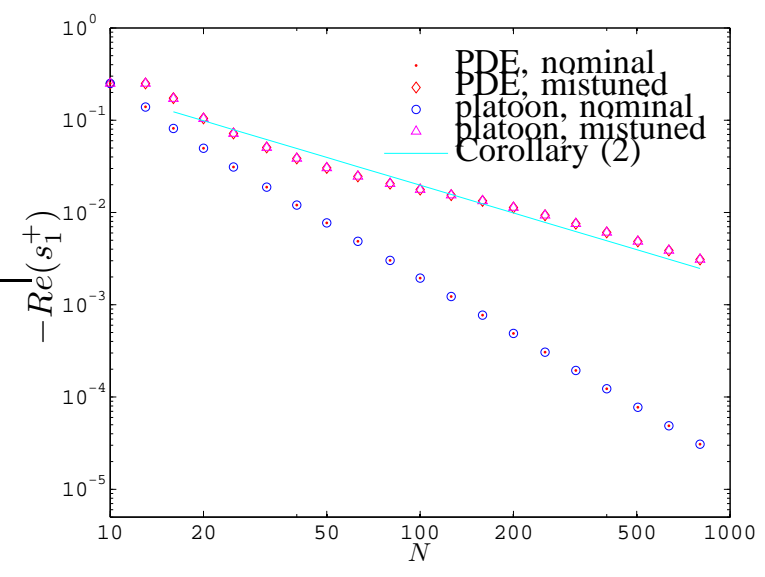

Fig. 2. The least stable eigenvalue of the closed loop platoon (i.e., of $A_{L-F}$ in (4)) and of the PDE (20) with Dirichlet boundary conditions, with and without mistuning, for a range of values of $N$. The nominal gains are $k_{0}=1$ and $b_{0}=0.5$. In the mistuned case, forward and backward controller gains are chosen as $k_{f}=k_{0}-0.1 \sin (x), k_{b}=k_{0}+0.1 \sin (x)$ (i.e., $k_{m}(x)=-\sin (x) / \sqrt{\pi}, k_{s}(x) \equiv 0$ and $\epsilon=0.2 \sqrt{\pi}$ ). For the platoon, controller gains are chosen by sampling the gains for the PDE as $k_{i}^{(f)}=1-0.1 \sin \left(y_{i}^{d}\right), k_{i}^{(b)}=1+0.1 \sin \left(y_{i}^{d}\right)$, where $y_{i}^{d}$ defined in (2) is the desired position of the $i^{\text {th }}$ vehicle. The legend "Corollary 2 " refers to the prediction by Corollary 2 . Note that the prediction of Corollary 2 is plotted only for $N>16$ to ensure that $1<<l_{c}$ (see (16)).

$\epsilon \rightarrow 0$, the optimal mistuning profile is given by $k_{m}^{*}(x)=$ $-\frac{\sin (x)}{\sqrt{\pi}}$. With this profile, the least stable eigenvalue is

$$
s_{1}^{+}(\epsilon)=-\frac{\epsilon \sqrt{\pi}}{2 b_{0}} \frac{1}{N}
$$

in the limit as $\epsilon \rightarrow 0$ and $N \rightarrow \infty$.

This corollary shows that even an arbitrarily small perturbation $\epsilon$ on the mean gain $k_{0}$ can greatly increase the closed loop platoon stability margin: with mistuning the least stable eigenvalue decays only as $O\left(\frac{1}{N}\right)$ instead of as $O\left(\frac{1}{N^{2}}\right)$, as it does without mistuning.

Figure 2 presents numerical validation of this prediction as well as the $\frac{1}{N^{2}}$ decay predicted for the nominal case in (17). The figure shows that

1) the platoon eigenvalues match the PDE eigenvalues accurately over a range of $N$, in both the nominal and mistuned cases, and

2) the mistuned eigenvalues show an order of magnitude improvement over the nominal case even though the controller gains differ from their nominal values only by $\pm 10 \%$.

For comparison, the figure also depicts the asymptotic eigenvalue formula given in Corollary 2.

Proof of Theorem 1. In order to compute the eigenvalues, we take a Laplace transform and consider a boundary value problem along the coordinate $x$ :

$-a_{0}^{2} \frac{\partial^{2} q}{\partial x^{2}}+s^{2} q+b_{0} s q=\epsilon\left[\frac{1}{2 \rho_{0}^{2}} \frac{\partial^{2}\left(k_{s} q\right)}{\partial x^{2}}+\frac{1}{\rho_{0}} \frac{\partial\left(k_{m} q\right)}{\partial x}\right]$.

Here $q(x)$ is the Laplace transform of $\tilde{\rho}(x, t)$ which satisfies Dirichlet boundary conditions: $q(0)=q(2 \pi)=0$. We are 
interested in eigenvalues of this boundary value problem, i.e., values of $s$ for which a solution to the homogeneous pde (21) exists with Dirichlet boundary conditions. To compute these, we use a perturbation method expressing the eigenfunction and eigenvalue in a series form:

$$
\begin{aligned}
q(x) & =p_{0}(x)+\epsilon p_{1}(x)+o(\epsilon), \\
s & =r_{0}+\epsilon r_{1}+o(\epsilon) .
\end{aligned}
$$

We note that $\epsilon r_{1}$ denotes the perturbation to the nominal eigenvalue $r_{0}$ as a result of the mistuning. Substituting Eq. (23) in Eq. (21) and doing an $O(1)$ balance, we get

$$
O(1): \quad-a_{0}^{2}\left(p_{0}\right)_{x x}+r_{0}^{2} p_{0}+b r_{0} p_{0}=0,
$$

whose eigen-solution is given by $p_{0}=d_{l} \sin \left(\frac{l x}{2}\right), r_{0}=s_{l}^{ \pm}$, where $l$ is an integer and $d_{l}$ is arbitrary real constant. Next,

$$
\begin{aligned}
& O(\epsilon): \quad-a_{0}^{2}\left(p_{1}\right)_{x x}+\left(r_{0}^{2}+b r_{0}\right) p_{1}= \\
& \quad \frac{k_{m}}{\rho_{0}} \frac{\partial p_{0}}{\partial x}+\frac{1}{2 \rho_{0}^{2}} \frac{\partial\left(k_{s} \frac{\partial p_{0}}{\partial x}\right)}{\partial x}-\left(2 r_{0} r_{1}+b_{0} r_{1}\right) p_{0}:=R
\end{aligned}
$$

Substituting $r_{0}=s_{l}^{ \pm}$(from Lemma 1) and $p_{0}=\sin \left(\frac{l x}{2}\right)$ on the left hand side leads to a resonance condition for the right hand side term, denoted by $R$. In particular for a solution $p_{1}$ to exist, $R$ must lie in the range space of the linear operator

$$
\left(-a_{0}^{2} \frac{\partial^{2}}{\partial x^{2}}+\left(r_{0}^{2}+b_{0} r_{0}\right)\right)
$$

For this self-adjoint operator, the range space is the complement of its null space $\left\{\sin \left(\frac{l x}{2}\right)\right\}$. This gives a resonance condition $\frac{1}{\pi}<R, \sin \left(\frac{l x}{2}\right)>=0$, where $<\cdot, \cdot>$ denotes the standard inner product in $L^{2}(0,2 \pi)$. Explicitly,

$$
\begin{aligned}
\left(2 r_{0}+b_{0}\right) r_{1}=\frac{l}{4 \pi \rho_{0}} & \int_{0}^{2 \pi} k_{m}(x) \sin (l x) d x \\
& -\frac{l^{2}}{8 \pi \rho_{0}^{2}} \int_{0}^{2 \pi} k_{s}(x) \cos ^{2}\left(\frac{l x}{2}\right) d x .
\end{aligned}
$$

For values of $r_{0}=s_{l}^{ \pm}$, this leads to an expression for perturbation in the two eigenvalues. We denote these perturbations as $r_{1}^{ \pm}$. For $r_{0}=s_{l}^{+}$, we have $b_{0}>>\left|2 r_{0}\right|$, so that

$$
r_{1}^{+}=\frac{l}{4 \pi \rho_{0} b_{0}} \int_{0}^{2 \pi} k_{m}(x) \sin (l x) d x+O\left(\frac{1}{N^{2}}\right) .
$$

Notice that in arriving at (25), we have dropped the integral involving $\frac{1}{\rho_{0}^{2}}$, since $\frac{1}{\rho_{0}^{2}}=O\left(\frac{1}{N^{2}}\right)$. Putting the formulas for eigenvalue perturbation (25) in (23), we get

$$
\begin{aligned}
s_{l}^{+}(\epsilon) \approx s_{l}^{+}(0)+\epsilon \frac{l}{4 \pi b_{0} \rho_{0}} \int_{0}^{2 \pi} k_{m}(x) \sin (l x) d x & \\
& +O\left(\epsilon^{2}\right)+O\left(\frac{1}{N^{2}}\right) .
\end{aligned}
$$

Since $s_{l}^{+}(0)=O\left(\frac{1}{N^{2}}\right)$ for $l<l_{c}$ (Corollary 1) and $\rho_{0} \approx$ $\frac{N}{2 \pi}$, we get the result. Although the perturbation $r_{1}^{-}$will be positive and subtract damping, it does so to the more stable eigenvalue $r_{0}^{-}$, so the less stable eigenvalues are not affected by it.

\section{CONCLUSION}

We developed a PDE model of a controlled vehicular platoon by modeling the motion of vehicles in a highway as fluid flow in a channel. By analyzing this PDE we explained the progressive loss of closed loop stability with increasing number of vehicles, when all vehicles use identical controllers. Further analysis showed how to improve closed loop stability by small amounts of mistuning. We proved and numerically validated that with arbitrary small amounts of mistuning, closed loop stability can be vastly improved.

The PDE developed in the paper can serve as a useful tool for further analysis and control-design for large platoons. We believe that similar PDEs can be developed and fruitfully exploited for a large number of problems involving multi-agent systems, not just vehicular platoons.

\section{REFERENCES}

[1] M. R. Jovanović and B. Bamieh, "On the ill-posedness of certain vehicular platoon control problems," IEEE Transactions on Automatic Control, vol. 50, no. 9, pp. 1307 - 1321, September 2005.

[2] P. Seiler, A. Pant, and K. Hedrick, "Disturbance propagation in vehicle strings," IEEE Transactions on Automatic Control, vol. 49, pp. 1835-1841, October 2004.

[3] S. S. Stankovic, M. J. Stanojevic, and D. D. Siljak, "Decentralized overlapping control of a platoon of vehicles," IEEE Transactions on Control Systems Technology, vol. 8, pp. 816-832, September 2000.

[4] P. Barooah and J. P. Hespanha, "Error amplification and distrubance propagation in vehicle strings," in Proceedings of the 44th IEEE conference on Decision and Control, December 2005.

[5] S. K. Yadlapalli, S. Darbha, and K. R. Rajagopal, "Information flow and its relation to the stability of the motion of vehicles in a rigid formation," in Proceedings of the 2005 American Control Conference, June 2005, pp. 1853-1858.

[6] S. Darbha, K. Hedrick, C. C. Chien, and P. Ioannou, "A comparison of spacing and headway control laws for automatically controlled vehicles," Vehicle System Dynamics, vol. 23, pp. 597-625, 1994.

[7] D. Helbing, "Traffic and related self-driven manyparticle systems," Review of Modern Physics, vol. 73, pp. 1067-1141, 2001.

[8] P. G. Mehta, G. Hagen, and A. Banaszuk, "Symmetry and symmetry-breaking in wave equation with feedback," SIAM Journal of Applied Dynamical Systems (accepted for publication), 2006.

[9] A. J. Rivas-Guerra and M. P. Mignolet, "Local/global effects of mistuning on the forced response of bladed disks," Journal of Engineering for Gas Turbines and Power, vol. 125, pp. 1-11, 2003. 